\title{
Improved Preisach -DOK Modeling on the Henkel Plot of A Longitudinal Barium Ferrite
}

\author{
Zhao Jing, Xue Fang, Han Ning \\ Department of Physics and Electrical Engineering \\ Handan College \\ Handan, 056005, China \\ 51547969@qq.com
}

\begin{abstract}
The Henkel plot and the $\Delta \mathrm{m}$ plot of a longitudinal barium ferrite hard disk and $\gamma$-Fe2O3 flexible disk were simulated by the improved Preisach-DOK model.The study results show that the barium ferrite exhibit large positive interactions, as shown by large positive in the $\Delta \mathrm{m}$ plot, and tend to resist demagnetization, leading the oriented barium ferrite possess the unusual remanence characteristic.
\end{abstract}

\section{Keywords-Preisach model, susceptibility, remanence}

\section{INTRODUCTION}

In recent years, the areal density of hard disk drives has doubled every year $[1,2]$. In general, the magnetic properties usually are derived from the ordinary hysteresis loop of the media, but this can lead to inconsistent results because the ordinary hysteresis loop includes both the reversible and the irreversible components of magnetization reversal, whereas the recording process depends only on the irreversible components, which are reflected only in the remanence loops. Reference [3] propose that the remanence loop parameters are more relevant to the magnetic recording process than the hysteresis loop parameters. At present, the development of the $\gamma-\mathrm{Fe} 2 \mathrm{O} 3$ media and the $\mathrm{CoFe}$ media have been restricted because of their bad remanence, thermal stability and corrosion resistance. Metal magnetic particles possessed of the larger recording potential since they have the higher coercivity and remanence, but there are two shortcomings: obvious corrosion resistance and the conditionality of particle dimension. The Barium ferrite media were studied for high density magnetic recording media, since they have good chemical stability and mechanical properties. In addition, their large magnetocrystalline anisotropy and lowmedia noise characteristics make it attractive for highdensity magnetic recording applications [4]. Especially, the Barium ferrite media have the more perfectly remanence characteristic so that they are applicable not only the coating vertical magnetic recording media but also the longitudinal recording media and the arbitrary oriented media. Reference [5] reported that they were studying the experiment of the coating vertical barium ferrite hard disk sample, however, this sample was not applied widely owing to the poor remanence characteristic.
In this study, we simulated the hysteresis loop and the Henkel plot and the $\Delta m$ plot of the BaFe and the $\gamma$ Fe2O3, and calculated the remanence properties.

\section{THE IMPOVED DOK MODEL}

The classical Preisach model can simulate only an irreversible magnetization [6], however, for the realistic media, the Preisach model must be improved so that it can simulate not only the irreversible magnetization but also the reversible magnetization. The E. D. Torre, J. Oti and G. Kàdàr (DOK) model [7] starts with a moving Preisach model and adds a magnetization-dependent calculation of reversible magnetization as well. Traditionally, the irreversible magnetization is calculated by integrating over the entire Preisach model plane. In this paper, an alternative method is to use a differential equation approach, working with susceptibility directly [8, 9].

In the model, the magnetization of a material $\mathrm{M}$ give the applied field $\mathrm{H}$. The operative field $\mathrm{h}$ which is defined as

$$
h=H+\alpha M \text {, }
$$

where $\alpha$ is the experimentally determined moving parameter. The total magnetization is calculated by simultaneously solving the equations below:

$$
\begin{aligned}
& \frac{d M}{d h}=\chi\left(h, M, m_{l}\right) \\
& \frac{d m_{I}}{d h}=\chi_{I}\left(h, M, m_{I}\right)
\end{aligned}
$$

In these equations, $\chi_{\text {is the total susceptibility, }} \chi_{I}$ is the irreversible susceptibility, $\mathrm{mI}$ is the normalized irreversible magnetization. If we consider the reversible magnetization, the total magnetization is the sum of its irreversible and reversible parts

$$
M / M_{S}=S m_{I}+(1-S) m_{R}
$$

where MS is the saturation magnetization, $S$ is the squareness of a media, $m_{R}$ is the normalized reversible magnetization, respectively. Taking the derivative with respect to the operative field

$$
\chi=d M / d h=S M_{S} \chi_{I}+(1-S) M_{S} \chi_{R}
$$


where $\chi_{R}$ is the reversible susceptibility.

For magnetic recording media, the Preisach distribution function is Gaussian and can be expressed as

$$
p\left(h_{i}, h_{c}\right)=\frac{1}{2 \pi \sigma_{i} \sigma_{c}} \cdot \exp \left\{-\frac{1}{2}\left[\frac{\left(h_{c}-h_{c i}\right)^{2}}{\sigma_{c}^{2}}+\frac{\left(h_{i}\right)^{2}}{\sigma_{i}^{2}}\right]\right\}
$$

where $h_{c i}$ is the average critical field, $h_{i}$ is the interaction field, $h_{c}$ is the critical field, $\sigma_{i}$ and $\sigma_{c}$ are the standard deviations of the interaction field and critical field, respectively. In this model, the irreversible susceptibility is different when the operative field $h$ is increasing or decreasing. When $h$ is increasing, we obtain the irreversible susceptibility

$$
\chi_{I, \text { inc }}=\frac{2}{\sigma \sqrt{2 \pi}} \exp \left[-\frac{\left(h-h_{c i}\right)^{2}}{2 \sigma^{2}}\right] \cdot \operatorname{erf}\left[\frac{h(1+\lambda)+h_{c i}(1-\lambda)}{\tau}\right]
$$

where

$$
\begin{aligned}
& \sigma=\sqrt{\sigma_{i}^{2}+\sigma_{k}^{2}}, \tau=\frac{2 \sigma_{i} \sigma_{k}}{\sigma}, \\
& \lambda=\frac{\left(\sigma_{k}^{2}-\sigma_{i}^{2}\right)}{\sigma^{2}} \text { and } \kappa=\frac{2 \sigma_{i}^{2}}{\sigma^{2}}=1-\lambda .
\end{aligned}
$$

For small values of $\sigma_{c}$, when $h_{c i}$ is much larger than $\sigma_{c}$, as is the case in recording media, we have

$\operatorname{erf}\left[\frac{h(1+\lambda)+h_{c i}(1-\lambda)}{\tau}\right] \approx 1$

giving

$\chi_{I, \text { inc }}(h)=\frac{2}{\sigma \sqrt{2 \pi}} \exp \left[-\frac{\left(h-h_{c i}\right)^{2}}{2 \sigma^{2}}\right]$

When $h$ is decreasing, we may rewrite equation (7) as $\chi_{I, \text { dec }}(h)=\frac{2}{\sigma \sqrt{2 \pi}} \exp \left[-\frac{\left(h+h_{c i}\right)^{2}}{2 \sigma^{2}}\right]$

For a state dependent the model, the reversible magnetization is

$$
m_{R}=a_{+} f(H)-a_{-} f(-H)
$$

where a good approximation is made by

$$
f(H)=1-e^{\gamma H} \text {. }
$$

According to the DOK model

$$
a_{+}=\frac{1+m_{I}}{2} \text { and } a_{-}=\frac{1-m_{I}}{2}
$$

The parameter $\gamma$ is another experimental-determined parameter related to the magnetic susceptibility. The use of this expression in equation (5) yields a full expression for the total operative susceptibility

$$
\chi=\frac{M_{S}\left\{\chi_{I}\left[\frac{1}{2}(f(H)+f(-H))(1-S)+S\right]+(1-S)\left(a_{+} f^{\prime}(H)-a_{-} f^{\prime}(-H)\right)\right\}}{\left[1+\alpha M_{S}(1-S)\left(a_{+} f^{\prime}(H)-a_{-} f^{\prime}(-H)\right)\right]}
$$

By the same token we can get system initial remanence magnetic

$\chi_{\mathrm{v}}=\frac{2}{\sigma \sqrt{2 \pi}} \exp \left(-\frac{\left(h-h_{c i}\right)^{2}}{2 \sigma^{2}}\right) \cdot\left(\operatorname{erf}\left[\frac{h+h_{c i}+\lambda\left(h-h_{c i}\right)}{\tau}\right]-\operatorname{erf}\left[\frac{h_{c i}+\lambda\left(h-h_{c i}\right)}{\tau}\right]\right)$

So the system magnetic susceptibility of initial magnetization curve is

$$
\chi_{i n i}=\frac{M_{S}\left\{\chi_{v}\left[\frac{1}{2}(f(H)+f(-H))(1-S)+S\right]+(1-S)\left(a_{+} f^{\prime}(H)-a_{-} f^{\prime}(-H)\right)\right\}}{\left[1+\alpha M_{S}(1-S)\left(a_{+} f^{\prime}(H)-a_{-} f^{\prime}(-H)\right)\right]}
$$

The total magnetization for input sequence of operative field can be computed at each step as

$$
M(h+\Delta h)=m(h)+\chi \cdot \Delta h
$$

where $\Delta h$ is the change in operative field at each step. The algorithm to calculate the total magnetization resulting from an input sequence of applied field using the fast DOK model is described in Table 1.

TABLE I. CALCULATING PROCEDURE OF IMPROVED DOK MODEL.

\begin{tabular}{|c|l|}
\hline Step & \multicolumn{1}{c|}{ Algorithm } \\
\hline $\mathbf{1}$ & Set $k=1, M_{1}=M_{0}, h_{1}=H_{1}+\alpha M_{1}$ and the convergence \\
tolerance $\mathcal{E}$
\end{tabular}

\section{RESUlTS AND DisCUSSION}

Fig. 1 shows the simulated hysteresis loop, which was compared with a measured hysteresis loop of the barium ferrite hard $\operatorname{disk}(\mathrm{BaFe})[10]$ and $\gamma$ - Fe2O3 flexible disk. The applied field sequence for $\mathrm{BaFe}$ was as following: Starting from +6000 Oe, which is sufficient to saturate the sample, the applied field was reduced to -1200 Oe. At -1200 Oe the field was reversed and increased to +1100 Oe, trancing a first-order reversal curve. At +1100 Oe the field was again reversed and reduced to -3000 Oe, tracing a second-order reversal curve. The applied field sequence for $\gamma-$ Fe2O3 was as following: Starting from +3000 Oe, which is sufficient to saturate the sample, the applied field was reduced to -400 Oe. At -400 Oe the field was reversed and increased to $+600 \mathrm{Oe}$, trancing a first-order reversal 
curve. At +600 Oe the field was again reversed and reduced to -2000 Oe, tracing a second-order reversal curve. As shown in Fig. 1, the model is in excellent agreement with the measured results throughout the process, including the second-order reversal curve, illustrating that the improved DOK model is applicable to a variety of magnetic recording media. The best-fit parameters are list in Table 2 .

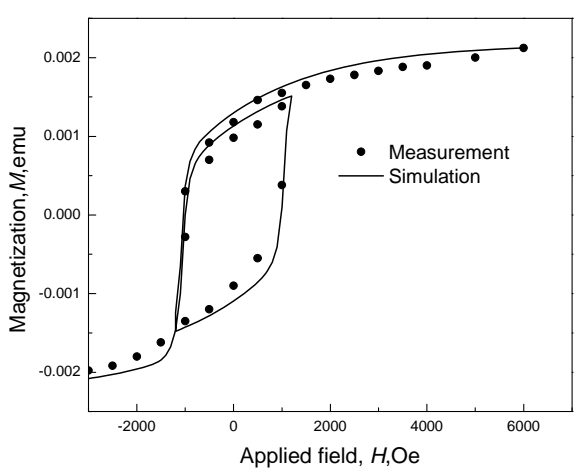

(a)

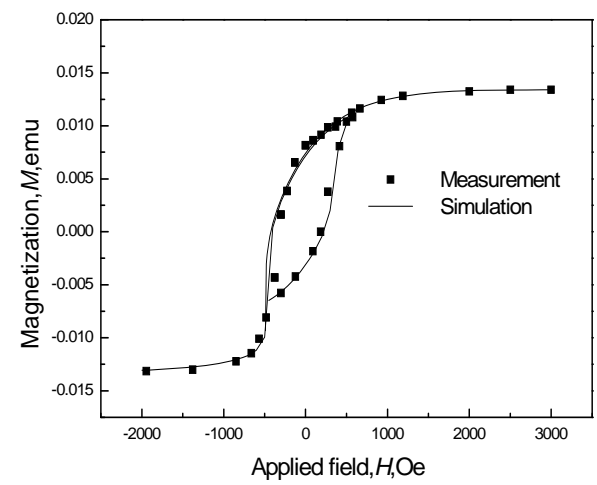

(b)

Figure 1. Measured(dot line)and simulated (solid line) hysteresis loop of the longitudinal BaFe hard disk sample (a) and the $\gamma$-Fe2O3 flexible disk sample (b) using the improved DOK model.

TABLE II. MODELING PARAMETERS OF THE LONGITUDINAL BAFE HARD DisK SAMPLE AND THE $\gamma$-Fe2O3 FLEXIBLE DISK SAMPLE.

\begin{tabular}{|c|c|c|}
\hline $\begin{array}{c}\text { Modeling } \\
\text { parameters }\end{array}$ & BaFe hard disk & $\gamma-\mathrm{Fe}_{2} \mathrm{O}_{3}$ flexible disk \\
\hline$M_{S}(\mathrm{emu})$ & 0.00212 & 0.0134 \\
\hline$S$ & 0.557 & 0.608 \\
\hline$\gamma$ & 0.000479 & 0.00169 \\
\hline
\end{tabular}

\begin{tabular}{|c|c|c|}
\hline$\alpha$ (Oe/emu) & 341963.7 & 23358 \\
\hline$h_{c i}(\mathrm{Oe})$ & 1267.03 & 522.28 \\
\hline$\sigma_{i}(\mathrm{Oe})$ & 14.66 & 16.205 \\
\hline$\sigma_{c}(\mathrm{Oe})$ & 407.11 & 130.808 \\
\hline
\end{tabular}
important role in the properties of magnetic recording media and are evident in the remanent processes. Comparison of the remanence processes shows small differences dependent on the initial magnetization state which can be directly attributed to interaction. First, starting from positive saturation, the field is removed and the media is brought to the saturation remanence $M_{\infty}$. The negative field $-\mathrm{H}$ is then applied and removed, and the remanence $\mathrm{Md}$ is measured. On the other hand, the system is initially in the demagnetized state, the positive field $\mathrm{H}$ is applied and then removed, and the remanence $\mathrm{Mr}$ is measured. In the absence of interactions, by considering the switching field distribution of the particles in the Preisach model, it can be show that Md and Mr are related by [11]

$$
\frac{\mathrm{M}_{\mathrm{d}}}{\mathrm{M}_{\infty}}=1-2 \frac{M_{r}}{M_{\infty}}
$$

The right of the equation is the Wohlfarth relation that it is expected to hold for an assembly of independent, singledomain, uniaxial partcles. The plot of $\mathrm{Md}$ as a function of $\mathrm{Mr}$ is known as the Henkel plot, and provides a common way to represent remanence properties. The various results obtained are partly modified by the presence of interactions. For any arbitrary Preisach distribution, the Henkel plot bounds is

$$
-\frac{\mathrm{M}_{\mathrm{r}}}{\mathrm{M}_{\infty}} \leq \frac{\mathrm{M}_{\mathrm{d}}}{\mathrm{M}_{\infty}} \leq 1-2 \frac{\mathrm{M}_{\mathrm{r}}}{\mathrm{M}_{\infty}}
$$

Because of the Preisach distribution with the factorized form $f\left(h_{c}\right) f\left(h_{i}\right)$ in this paper, the Henkel plot yield the following remanence law:

$$
\frac{\mathrm{M}_{\mathrm{d}}}{\mathrm{M}_{\infty}}=1-2 \sqrt{\frac{M_{r}}{M_{\infty}}}
$$

This remarkably simple law is completely independent of $f(x)$. Particle-particle or wall-wall interaction effects can be put in evidence by plotting $M_{d} / M_{\infty}$ vs $M_{r} / M_{\infty}$ (Henkel plot) and by looking at any departure from the Wohlfarth relation. Deviations of the measured Henkel plots from the straight line representing Eq. ( 19) have been interpreted as indications of " positive" and " negative" interactions depending on whether the measured 
variation is " above" or " below" the line representing the Wohlfarth relation.

The difference between the curves can be measured by

$$
\Delta m=M_{d}(H)-\left(1-2 M_{r}(H)\right)
$$

Plots of $\Delta m$ vs $\mathrm{H}$ have come to be known as $\Delta m$ plots. $\Delta m$ plots as well as Henkel plots can reflect the interaction effect among neighboring particles. $\Delta m>0$, it Show that for positive interaction; $\Delta m<0$, Show that for negative interaction.

For researching the interactions between particles of the $\mathrm{BaFe}$, we simulated the Isothermal remanent magnetization curve, and then obtained the Henkel plot with the BaFe and the $\gamma$-Fe2O3, shown in Fig. 2. According to the Eq. ( 22), we drawed the $\Delta m$ plots, shown in Fig. 3.

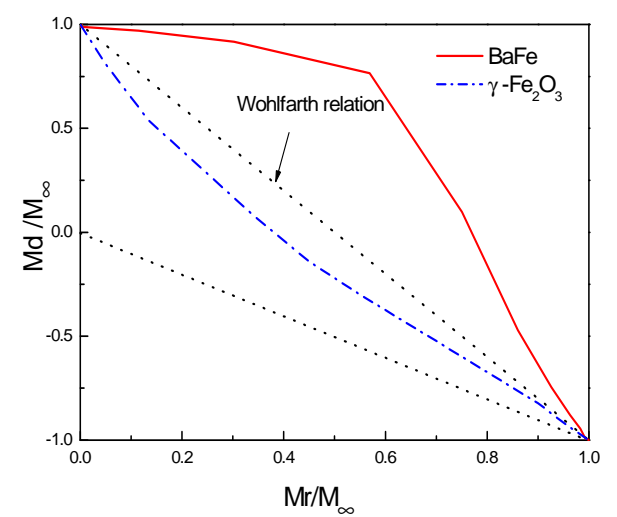

Figure 2. Henkel plots for the $\gamma_{\text {-Fe2O3 flexible disk sample (dash dot }}$ line) and longitudinal BaFe hard disk sample (solid line).

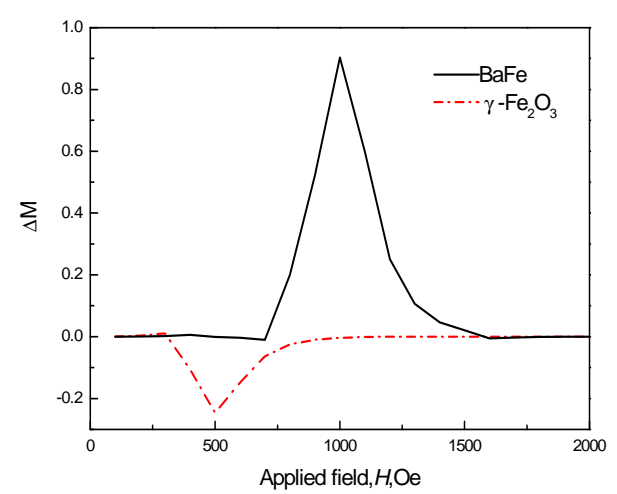

Figure 3. $\Delta m$ plots for the $\gamma$-Fe2O3 flexible disk sample (dash dot line) and longitudinal BaFe hard disk sample (solid line).
We observed the Henkel plot and the $\Delta m$ plots of the BaFe has larger departure from the Wohlfarth relation and there is a big positive peak in the $\Delta m$ plots, in other words, the interactions between particles of the BaFe are stronger than of the $\gamma$-Fe2O3, making for the BaFe have the more perfectly remanence characteristic. The BaFe has the large magnetocrystalline anisotropy and its particle shape has not many cavities and branches so that it is more perfect than of the acicular $\gamma$-Fe2O3. One of the possible reasons that the remanence properties of the BaFe are better than of the $\gamma$ Fe2O3 is that the coercivity of acicular $\gamma-$ Fe2O3 magnetic particle is lie on the shape anisotropy. With the fineness and uniformity of particles, we can enhance its coercivity. The $\mathrm{BaFe}$ has the large magnetocrystalline anisotropy and its particle shape has not many cavities and branches so that it is more perfect than of the acicular $\gamma$ - Fe2O3, leading the oriented Bafe possess the unusual remanence characteristic.

\section{CONCLUSIONS}

We apply a improved DOK model of hysteresis to the remanence properties of a longitudinal barium ferrite hard disk and a $\gamma-$ Fe2O3 flexible disk. The Preisach model decomposes the magnetization process into reversible and irreversible parts, and decomposes the irreversible part into many magnetic units with moment $\mu_{0}$, coercivity $h_{c}$ and bias field $h_{i}$. In the improved DOK model, it is necessary to model the reversible component separately and add it proportionally to the irreversible component in order to calculate the total magnetization. Many magnetic processes of a magnetic material are simulated by different susceptibility functions. The reversal curve, the hysteresis loop, the reversible curve, the Henkel plot and the $\Delta m$ plot of a longitudinal barium ferrite hard disk and $\gamma-\mathrm{Fe} 2 \mathrm{O} 3$ flexible disk were simulated by the improved DOK model, and we get some parameters. The study results show that the parameters not only embody the varied magnetic processes but also reflect the remanence properties of the two samples.

In the study of the longitudinal barium ferrite hard disk and $\gamma$-Fe2O3 flexible disk, the coercivity of the barium ferrite decided by the magnetocrystalline anisotropy is larger than that of the acicular $\gamma$-Fe2O3 determined by the shape anisotropy. By the analysis of the Henkel plot and the $\Delta m$ plot, the barium ferrite exhibit large positive interactions, as shown by large positive in the $\Delta m$ plot, and tend to resist demagnetization, leading the oriented barium ferrite possess the unusual remanence characteristic.

\section{REFERENCES}

[1] Coffey K R, Thomson T, Thiele J U. J. Appl. Phys., 2002; 92(8): 4553

[2] Speliotis D E. J. Mag. Mag. Mater., 1999; 193: 29

[3] Speliotis D E. J. Appl. Phys., 1990; 67: 5358 
[4] Kakizaki K, Taguchi H, Hiratsuka N. J. Mag. Mag. Mater., 2004; 272-276: 2241

[5] Simmons R G. IEEE Trans. Magn., 1990; 26: 93

[6] Preisach F. Z. Phys., 1935; 94: 277

[7] Torre E D, Oti J, Kàdàr G. IEEE Trans. Magn., 1990; 26: 3052
[8] Reimers A, Torre E D. IEEE Trans. Magn., 1998; 34: 3857

[9] Patel U D, Torre E D. IEEE Trans. Magn., 2002; 38(2): 869

[10] Torre E D. IEEE Trans. Magn., 1994; 30: 4987

[11] Bertotti G, Basso V. J. Appl. Phys., 1993; 73(10): 5827 